\title{
¿Quién teme a la psicología feminista? Reflexiones sobre las construcciones discursivas de profesores, estudiantes y profesionales de psicología para que cuando el género entre en el aula, el feminismo no salga por la ventana
}

Teresa Cabruja Ubach *

Resumen: En el texto se problematizará la inclusión del género en psicología a partir de las construcciones discursivas obtenidas en una investigación cualitativa sobre las opiniones de estudiantes, profesores y profesionales. Dado que la mayor aceptación asimila "género" a "diferencia sexual" desde la tradición positivista y dualista, se neutraliza su capacidad transformadora. Esta versión "descomprometida” del género, se disocia discursivamente del feminismo social y las epistemologías feministas que generan mayores resistencias y temores. Tal y como argumentaré, una psicología positivista con su supuesta neutralidad y objetividad así como la permanencia de un imaginario androcéntrico y etnocéntrico dificultan la capacidad de cuestionar una psicología poco sensible a las relaciones de poder. Finamente, propongo la necesidad de combinar un análisis del currículo oculto con una comprensión de las argumentaciones y creencias que forman parte de la vida cotidiana del ejercicio y docencia de la psicología. Así se pueden retomar de forma conjunta los mecanismos de (re)producción y difusión de la ciencia con las relaciones e interacciones académicas y profesionales marcadas por el sistema sexo-género, que operan de forma compleja en las combinaciones de ciencia e ideología o discurso y acción.

Palabras clave: género, psicología, epistemología feminista crítica, relaciones de poder, postmodernidad, subjetividad

\section{Who is afraid of feminist psychology? \\ Reflections on teachers', students' and professionals' discoursive constructions to prevent the exclusion of feminism from classrooms as gender comes in}

Abstract: This text provides a discussion on the inclusion of gender in psychology from the discoursive constructions obtained in a qualitative investigation about students', teachers' and professionals' opinions. Since most people who support this idea relate "gender" to "sexual

* Profesora Titular Psicología Social. Departamento de Psicología. Universidad de Girona. España. teresa.cabruja@udg.es 
difference", based on to the positivist and dualistic tradition, its capacity of transformation is neutralized. In discoursive terms, this "uncommitted" version of gender is dissociated from social feminism and the feminist epistemologies that generate great resistance and fear. As it will be explained, a positivist kind of psychology with supposed neutrality and objectivity, as well as the continuous character of an androcentric and ethnocentric imaginary field, makes it difficult to question a kind of psychology that is little sensitive to power relations. Finally, there is a proposal of combining the analysis of a hidden curriculum with the understanding of argumentation and beliefs that consist part of everyday teaching and working with psychology. This way, it is possible to resume the mechanisms of science (re)production and diffusion with the academic and profesional relations and interactions that are characterized by the sexgender system and operate discourse and action in a complex way, in the combinations of science and ideology.

Key words: gender; psychology; critical feminist epistemology; power relations; post-modernity; subjectivity.

\section{Introducción: las dilemáticas acepciones del género y la resistencia a la psicología feminista}

Antes de introducir los apartados del siguiente artículo, me gustaría comentar brevemente las razones que me llevaron a realizar un estudio sobre las opiniones por parte de compañer@s de trabajo, de estudiantes o de profesionales de la psicología respecto a las posibilidades de incorporar los estudios de género, las epistemologías feministas y la recuperación de las mujeres psicólogas de su historia. El proyecto en su globalidad, incluyó una investigación de documentos y recursos disponibles sobre Psicologia y género, Psicologia y Feminismo y Psicologia de la mujer en Girona (ciudad y provincia de Cataluña, España) y la realización de una web de recursos e intercambio entre profesionales, asociaciones, investigadores y estudiantes. Y, especialmente un estudio empírico con metodología cualitativa sobre ¿Cómo incorporar los estudios feministas, de género y las mujeres de la historia de la psicologia en la formación psicológica? (Cabruja et al, 2004) ${ }^{1}$, que presentaré muy brevemente en el siguiente apartado antes de comentar algunos de sus resultados. ¿Qué se entendía por cada una de estas denominaciones ${ }^{2}$ ? ¿qué opiniones había respecto a su incorporación

1. El estudio Com incorporar les dones psicòlogues i els estudis feministas a la formació psicológica? Estudi pilot a Girona i comarques fue financiado por el Institut Català de les Dones (2004) y, posteriormente, por una ayuda a la dinamización y mantenimiento de la actividad investigadora de la Universidad de Girona (2005). Contó con la participación temporal en sus distintas fases de realización del@s siguientes profesora/es, estudiantes en prácticum e investigadores/as doctorales: Pilar Albertin; Antonia Dorado, Laura Espinas, Eduard Juanolay Anna Torrent, a quienes agradezco sus diversas colaboraciones.

2. Aquí me he referido a "género", pero justamente las diferencias y semejanzas entre "psicología de la mujer", "género" y "psicología feminista" no necesariamente coinciden, tal y como desarrollaré en el texto. 
en la formación? ¿se consideraban necesarias?, ¿para qué? Y ¿ por qué sí? o ¿por qué no? Y, suponiendo que sí: ¿de qué manera incluirlas?

Lo que me interesaba explorar era qué posiciones y argumentos se podían encontrar respecto al tema del género, en un momento en el que se inician actuaciones sobre la violencia doméstica, el acoso sexual, la igualdad, etc. Género y Psicologia. Pues, si bien el debate en sí, ya se ha ido desarrollando en el interior del feminismo, durante la última mitad del siglo XX, respecto a "integración curricular" o "estudios especializados o específicos", actualmente se prolonga en cuanto a la perspectiva de género, así cómo respecto al eurocentrismo y occidentalismo del currículo en los estudios y la necesidad de interconectar la crítica de etnocentrismo, con las de logocentrismo, colonialismo, heterosexismo y patriarcalismo.

Pero, además de conocer cómo se construyen estas dinámicas en el habla cotidiana, otro interés, era el de conectar las aportaciones de distintas epistemologías feministas de la ciencia, en la línea de autoras como Sandra Harding, Evelyn Fox-Keller, Hellen Longino y sus explicaciones respecto al androcentrismo y sexismo de la ciencia y cómo transformarlo, en su especifica manifestación en el campo de la Psicología. Junto con aportaciones como las de Donna Haraway y las propuestas de "conocimiento situado", además de otras críticas del feminismo postmoderno y el postfeminismo, que se están adaptando a los retos de psicología socioconstruccionista y crítica. La Psicología, constituye, según como, un caso especial, pues a diferencia de otras disciplinas como la sociología, la antropología o la historia, por ejemplo, donde más a menudo se han dado intentos de combinar el paradigma científico con el compromiso político y la incuestionable alusión a cuestiones como la clase, las sexualidades y el racismo, las resistencias han sido mucho mayores. Una de las principales razones, por supuesto, es debida a que la tendencia dominante sea su afán por identificarse y pretenderse una ciencia experimental (con la idea de la psicología como neutra, objetiva y universal). Incluso respecto a una acción aparentemente más simple y que no se contradeciría con la psicología positivista que consiste en reconocer e incorporar a las mujeres que han desarrollado aportaciones de gran relevancia en su historia. Tal y como constatan para las pioneras anglosajonas, autoras como Rhoda Unger, Jane Marecek y Hare-Mustin en diversas publicaciones o, más recientemente, con la revisión de García Dauder

3. En este sentido, algunos textos en el estado español constituyen una buena referencia para este tipo de debate. Uno de ellos, es el que escribió Malesús Izquierdo, en 1988 , titulado "iSon las mujeres objeto de estudio para las ciencias sociales?" p.51-66, Papers Revista de Sociología. Barcelona: UAB, sobre el dilema de estudiar la desigual dad sexual incorporando un concepto de género y otro es el de Verena Stölcke, también en 1988 debatíendo sobre la utilidad de estudios específicos (Stölke, | 988). 
(2005), para las psicólogas españolas de la república y el exilio, el interesante trabajo de García-Colmenares (2006). Más difícil aún, tal y como explicaban Bhavnani y Phoenix (1994) en su Introducción a Shifting Identities/Shifting Racisms. A feminism \& Psychological Reader (Bhavnani; Phoenix, 1994), para el cruce de feminismo, acción colectiva, academicismo y racismo.

De todas formas, aunque la psicología feminista, se ha ido desartollando de distintas maneras, unas veces por mujeres que han ido compartiendo la marginalización otras como proyecto colectivo (Unger, 1998), considero que continúa siendo bastante difícil denunciar los mecanismos institucionales que dificultan su integración junto los más sutiles de discriminación. Tal y como Hare-Mustin y Marecek (1990) afirman:

La psicología tradicional se mantuvo de hecho sin mujeres en otro sentido. [...] Las pocas que había a menudo se volvían invisibles o quedaban marginadas, no se les reconocía su trabajo o se les negaban los recursos para una actividad académica productiva " $y$ aunque "Un remedio para la invisibilidad de las mujeres en psicología ha consistido en centrar de nuevo la atención en las aportaciones y logros de las mujeres excepcionales. Esto constituye un avance importante, pero sólo es algo parcial, ya que no pone en discusión de modo eficaz la norma masculina de la psicología sin mujeres (Hare-Mustin; Marecek, 1990, p.22-23).

Claro que también hay resistencias de la disciplina por su fuerte inscripción en la racionalidad moderna positivista hacia otras perspectivas más o menos críticas con sus propios fundamentos a lo largo de la historia. De las corrientes menos positivistas como pueden ser el interaccionismo simbólico o el psicoanálisis (este "otro" de la psicología) y de los temores de su "contaminación" al proyecto positivista, dan buena cuenta el caso de las más históricas. Pero que se da, también, con la psicología postmoderna, discursiva o socio construccionista. Es decir, en las relaciones de la psicología "mainstream" con la "psicología critica" (que tampoco es una etiqueta que unifique o en donde se reconozcan la variedad de psicólogos y psicólogas que, desde aproximaciones distintas, intentan cuestionar conceptos, técnicas y actuaciones de la psicología. Todas ellas continúan siendo bastante "marginalizadas" como argumentan Prillelstensky y Fox (1997), aún a pesar de la necesidad que se puso en evidencia con la crisis de finales de los setenta del pasado siglo, de una psicología crítica "que intente debilitar los efectos de poder de la ciencia y de generar prácticas que no reproduzcan las estructuras de dominación”, parafraseando a Ibáñez (1997). Y de la necesidad de los compromisos ético-políticos y del carácter político de la psicología o su ideología, como se ha ido desarrollando en América Latina por parte de la psicología de la liberación (Martín-Baró, 1987), la comunitaria 
(Montero, 1994; Spink; Spink, 2006), la colectiva (Fernández-Christlieb, 1994) o, más recientemente, por las aportaciones postcoloniales. Además, por supuesto, que deberíamos preguntarnos tanto hasta qué punto podemos hablar de psicología crítica (Cabruja; Gordo, 2001), cómo qué supone esta inclusión y sus esfuerzos de legitimación (Gordo, 2002) o respecto a la institucionalización del socio construccionismo, como plantea Íñiguez (2005) para el caso específico de la psicología social, tal y como ha ido sucediendo para la psicología feminista. A estos dos grandes temas se añaden el de las relaciones de poder académicas, no sólo en cuanto a la dificultad que han tenido estos currículos para integrarse en la docencia, para publicar, para ser reconocidos como estudios, por ejemplo, sino además la polémica, sobre si, según qué tipo de incorporación no los desprovee, justamente, de todo el potencial que los ha generado, en este intento de conectar la construcción de saberes y las prácticas políticas. Debate persistente en la historia del feminismo y que se amplia con la crítica realizada por el feminismo de la tercera ola a finales de los ochenta y el postfeminismo (Figueroa-Sarriera, Milagros-López y Román, 1994) , y que podemos hallar de forma parecida en el interior de los Estudios Queer, hacia las sexualidades heteronormativas, a la vez que se discuten las implicaciones de participar en la institucionalización del conocimiento y la posible pérdida de su subversión al salir de la calle (Córdoba et al., 2005. O como introducen Bell Hooks (1984) para el activismo feminista negro, Spivak (1988), con la posición de l@s estudios subalternos y su apropiación desde la academia asimismo como la dificultad de escapar de las lógicas de representación occidental, o Andalzúa (1987) con el“mestizaje”para 1@s estudios chican@s.

Como además el neoliberalismo conlleva un ensalzamiento del individualismo más feroz, es bastante fácil preguntarnos, en qué medida, otra vez, la psicología que ya tiene una tradición de ofrecer explicaciones bien individualistas de problemas de carácter social o político, manteniendo el status quo (Fox; Prilleltensky, 1996) o participando en la regulación social con los distintos regímenes de verdad en la línea de los trabajos desarrollados por Kenneth Gergen, Edward Sampson y Nicholas Rose, o generando efectos “involuntariamente autoritarios" (Ibáñez, 1994), recuperará el biologismo y el experimentalismo que la caracterizan con sus más sutiles formas de dominación patriarcal y racistas o etnocéntricas. Pero es que incluso desde gran parte de la psicología postmoderna o posestructuralista ha habido una gran dificultad en reconocer la influencia y participación de las aportaciones feministas respecto al cuestionamiento del sujeto, la reflexividad y la ideología o la misma metodología (Cabruja, 1996; Henwood et al., 1998).

Disponemos de diversos estudios que recogen testimonios respecto a la relación entre feminismo y psicología a partir de entrevistas, biografías o, in- 
cluso investigaciones sobre qué implica posicionarse como psicólogas feministas. En la misma presentación de una recopilación de textos en este sentido, publicada por Burman (1990), se hace constatar

la dificultad de llevar a la discusión pública, justamente lo que en la estructuración de lo público se ha suprimido. Estos temas son la marginalización del género (así como "raza", clase y sexualidad): tanto en los marcos teóricos ofrecidos por la psicología, como en la experiencia de las mujeres que están implicadas con la psicología-ya sea enseñando, investigando o en lo profesional- (Burman, 1990, p.1).

Lo que comparten estas reflexiones es justamente, un debate sobre cómo y de qué manera las feministas están posicionadas dentro o fuera de la psicología ortodoxa y las implicaciones de tales posicionamientos, con una interesante interpretación respecto a la separación entre teoría y práctica, en la cual se reproduce la separación mental y manual-aplicada y se irresponsabiliza a la teoría de las implicaciones que tiene para la investigación. Desde la sutil devaluación de trato y consideración de las mujeres en los departamentos de psicología (Kagan; Lewis, 1990) hasta cómo la retórica de la ciencia desplaza y excluye la investigación feminista y la lésbica en tanto las considera como políticas o partidistas (Kitzinger, 1990). Pero, experiencias y reflexiones parecidas las hallamos, también, en la más reciente investigación de Cressy, Harrick y Fuehrer (2002) sobre las identidades de psicólogas feministas desde una perspectiva de investigación narrativa y socio construccionista.

Asimismo, no podemos evitar el considerar también, tal y como previene Burman (1990), el tipo de inclusiones ${ }^{4}$ que se realizan, es decir, que

las maneras en las cuales algunos aspectos de la "investigación de género" ha sido incorporada en la psicología mainstream ilustra algunos de los dilemas y compromisos implicados en la lucha por el reconocimiento y el trabajo en el interior del sistema (Burman, 1990, p.7).

La cuestión es el particular estatus del feminismo en la psicología. Pero es que incluso en la llamada "psicología política" (en sus publicaciones y actos),

4. Y, en este sentido, el caso de la creación de la sección de la Psicología de la Mujer en la British Psychological Society que, por ejemplo, no existe en España como tal, ni en otros paises de la Unión Europea, no es ajena a los dilemas de una respuesta institucional de este tipo. En España se han realizado diversos estudios sobre el "curriculum oculto" en las distintas disciplinas o sobre el "techo de cristal". Sin embargo, me interesaba específicamente el caso de la psicología y directamente las opiniones argumentadas de psicólog@s y estudiantes. 
Capdevila y Unger (2006) hacen notar la poca atención que ha recibido la teoría feminista y que se han conformado como dos áreas separadas por ambas líneas y con grandes dificultades para quieres se encuentran en su intersección y mi experiencia fue parecida respecto a la psicología comunitaria.

Por eso me parece necesario priorizar además de los estudios sobre las mujeres psicólogas, la visibilización de los análisis de los procesos político-sexuales envueltos en la producción y la práctica de la psicología. De aquí no se deduce que los "psicólogos sean sexistas" sino más bien que una cultura y una ciencia androcéntricas permite y produce según qué tipo de comportamientos. Tensiones parecidas a las que Callaghan (2005) describe en el contexto sudafricano para abordar, justamente, la formación del "psicólogo profesional" Desde lo que puede ser discriminación más jerárquica o relacional por razón de sexo, hasta la misma resistencia en posiciones subordinadas, como estudiantes, o entrevistad@sousuari@s a lo que los trabajos feministas, más en la línea de la diferencia, han desarrollado como "autoridad" femenina. Estas posiciones están marcadas por las relaciones sexo-género y se insieren en un entramado discursivo que comporta una red de derechos, obligaciones y posibilidades de acción que repercuten en la subjetividad y las relaciones de poder. Donde se combinan prácticas discursivas distintas y como Walkerdine (1981) puso en evidencia en el análisis de una clase pueden trastocar ni que sea temporalmente, las relaciones de poder, en este caso: maestra-niños, aludiendo al discurso de la sexualidad, por ejemplo.

Para ello necesitaba explorar no sólo las opiniones de 1@s psicólog@s que están más o menos próximos a estas perspectivas, sino también las que había por parte de estudiantes, profesores y profesionales, que justamente no incluyen un enfoque de género. ¿Cuáles eran las ideas o imágenes hacia las relaciones entre psicología y género y una psicología feminista? ¿qué opiniones?, ¿qué argumentaciones y en función de qué?, Y, en este sentido constituye un estudio novedoso. La totalidad de los resultados (Cabruja et al., 2004), que no podré presentar en este artículo fueron muy variados, desde opiniones que lo veían sólo como una área más, a otras que lo identificaban con la psicología diferencial, o que veían necesario tener más formación al respecto porque la experiencia en la atención psicológica o la atención en maltratos llevaba a necesitar más elementos de comprensión o, al contrario, a rechazar, de una manera u otra, una gran parte de lo que se asimilaba a feminismo, aunque se pudiera dar apoyo, parcialmente, justamente a algunas de sus aportaciones.

De hecho, éste último aspecto es el que desarrollaré aquí, porque, en realidad, el hilo argumental del artículo coincide con lo que ya se formulaban hace 17 años atrás, Coulson y Bhavnani (1990, p.63) respecto a la necesidad de preguntarse: “¿Qué ha pasado y que continúa pasando con el feminismo, [...] 
para discutir algunos de los actuales problemas del enfoque feminista en psicología?”. Por supuesto que las razones que introduje anteriormente respecto a la problemática inclusión de los estudios feministas en la psicología por los dualismos del racionalismo positivista: la separación ciencia-ideología o, dicho de otra manera: objetividad- subjetividad, ciencia-política y, seguidamente, el hecho de que la mayoría de perspectivas feministas, desde los años 70 , excepto una, la del feminismo positivista (según la clasificación de Harding, 1986), que se centra más en sesgos e interpretaciones de resultados de una ciencia "mal hecha” más bien lo que hacen es transformar los conceptos, métodos y prácticas de la psicología.

Por eso me parecía interesante explorar cómo se están construyendo legitimaciones o no, de perspectivas alternativas a la ciencia positivista, y, sobretodo, en un momento de veloz transformación y reapropiación de las demandas de distintos grupos sociales. También, cómo se combina el haber sido socializados, ya no sólo en las relaciones y hegemonismo patriarcales, sino, también, en distintos tipos de resistencia a ellas. Es decir, con cierto conocimiento y/o participación directa o indirecta, de la transmisión de ideas del feminismo, de una cierta "aculturación feminista" tal como la entiende la antropóloga mexicana Marcela Lagarde (2001), como transmisión de prácticas y experiencias feministas en condiciones de hegemonismo patriarcal. Además, de la influencia de la crítica étnica y postcolonial, como los Estudios chicanos o los Subalternos por ejemplo (para el etnocentrismo y el colonialismo) o los Estudios Queer para la sexualidad, que se desarrollan de forma parecida y, a menudo, conjunta, respecto a los dilemas del compromiso y qué tipo de inclusión en la academia, tal y como comenté anteriormente.

No es tampoco ninguna casualidad que, incluso después del "Giro linguiístico o interpretativo" y de los múltiples post, muchas feministas se hayan orientado al psicoanálisis, entre otras razones por encontrarlo más flexible y de mayor ayuda para comprender temas sobre la feminidad, el deseo y por no ser tan tajante respecto a que la psicología feminista "no es psicología", en base a una definición "única" de la psicología como "positivista". De hecho, en los resultados de los grupos de discusión, en el momento de recordar mujeres que formaron parte de la historia de la psicología, se produjo una gran coincidencia en que los únicos nombres que se recordaban eran de mujeres psicoanalistas. Sin embargo, en el momento de explicar el porqué, se argumentaban explicaciones muy distintas, tal y como presentaré en la Reflexión sobre los resultados, aunque antes introduciré una breve descripción de la investigación. 
Breve descripción de la investigación sobre género, feminismo y psicología: muestra, método y análisis

La investigación empírica acerca de “¿Cómo incorporar los estudios feministas, de género y las mujeres psicólogas en la formación psicológica?” (Cabruja et al., 2004; 2005), dentro del proyecto general comentado anteriormente, consistió en recoger y discutir las opiniones con una muestra de estudiantes, investigadores y profesionales que se eligieron aplicando un criterio de homogeneidad (psicología) y varios de heterogeneidad que se describen seguidamente.

Se combinaron dos técnicas de recogida de datos de la metodología cualitativas: la entrevista en profundidad y los grupos de discusión, en base a lo que cada una de ellas puede aportar ${ }^{6}$. Se realizaron 8 entrevistas en profundidad (a 4 personas que ocupaban algún lugar en la gestión de los estudios e investigación en psicología en la universidad así como, 2 del colegio de psicólogos y 2 más a profesionales). Y 3 grupos de discusión: Dos grupos con profesionales y docentes de distinta orientación psicológica (cognitivoconductual, psicoanálítica, sistémica,...), distinta especialidad (clinica, jurídica, social, comunitaria, educativa,...), distintos sectores (público, cooperación/ asociacionista y privado), distintas comunidades y poblaciones (emigrantes, familias, adultos, infantil,...), con distintos grados de compromiso con el feminismo o los estudios de género y dedicados o a la investigación-docencia o a la intervención y un tercer grupo con estudiantes, también en distintas situaciones y niveles (desde licenciatura a doctorado). El total de la muestra fue de 29 personas: 7 hombres y 22 mujeres. El guión preparado tomaba en consideración que se hablara sobre conocimientos, explicaciones, opiniones y sugerencias respecto a su lugar en la formación, su necesidad y sus posibilidades o inconvenientes.

El primer análisis de todos los datos obtenidos se realizó básicamente de tipo categorial e interpretativo pero bastante sistematizado y exhaustivo para presentar las propuestas, inconvenientes y formas sobre las posibilidades que habían. Pero aquí sólo voy a referenciar unos cuantos ejemplos de construcciones discursivas sobre la psicología, el feminismo y el género. Tomando en

5. Por otra, parte, quería usar la misma metodología, para "movilizar" al go en relación con el tema y generar posibilidades, también, muy concretas de colaboración con Ong's, profesionales, etc. tanto de difusión como de discusión e intercambio y que se han traducido en una página web sobre recursosy una red.Agradezco la colaboración de tod@s $@$ s participantes en lainvestigación.

6. Ver por ejemplo los comentarios de lbáñez ( 1979 ) respecto a las posibilidades y limitaciones del grupo de discusión y los de dicados a la entrevista de Ruiz Olabuenagua e Ispizua ( | 989) respecto a la entrevista. 
consideración la idea de "posición" discursiva (Davies; Harré, 1990; Howllay, 1986) y del carácter dilemático de las argumentaciones en su construcción retórica (Billig et al., 1988). Me centraré más bien, en algunos patrones argumentativos o discursos que sostienen y posibilitan prácticas cotidianas y, a su vez, legitiman o justifican posiciones en las relaciones sociales que pueden implicar desigualdad o ser entendidas en términos de relaciones de poder. Se entienden los discursos, como prácticas sociales (Iñiguez, 2003; Parker, 1992; Potter, 1996) e ideológicas desde el feminismo postestructuralista (Hollway, 1989; Ramazanoglu; Holland, 2002; Wilkinson; Kitzinger, 1995), que resumo en varios aspectos: sobrepasan su dimensión puramente verbal o linguiística y tienen una participación activa tanto en el habla cotidiana como las practicas institucionales así como en la construcción de la intersubjetividad y las relaciones sociales. Vehiculan y combinan los procesos, discursos e imaginarios disponibles culturalmente, en el sentido de qué permiten, qué legitiman o qué autorizan, pero, a su vez, cómo hacen posibles distintas posiciones de sujeto. En consonancia con esta línea de análisis, recordar que no se toman las respuestas como indicadoras directas de las actitudes ${ }^{7}$ de las personas, sino, más bien, tal y como han desarrollado Wetherell y Potter (1992) como indicadores de posibilidades disponibles en el lenguaje, utilizables o reproducibles por distintos sujetos y por lo tanto, susceptibles de análisis respecto a lo que incorporan de ideas, justificaciones, imaginarios, simbólicos. A pesar de las críticas a este tipo de análisis, pues, efectivamente resulta bastante difícil explicitar objetivos y posiciones claramente, en el sentido de ¿qué constituye un "análisis feminista”? y ¿cómo explicitar más mi implicación? Comentar que se intentó incorporar la reflexividad de distintas maneras, pero quedando aún pendientes algunos aspectos $^{8}$.

Introduciré pues algunas de las creencias que movilizaron estas construcciones discursivas alrededor de los temas que planteé, para entenderlas respecto a qué efectos tienen o qué imaginarios movilizan, estas argumentaciones o ideas respecto a que mitos y estereotipos incorporan o, al contrario, desplazan para una comprensión de las relaciones de poder, en términos folucaultianos, que emergen más bien de un complicado sistema y redes de relaciones y acciones

7. Dentro de una misma entrevista, hecha a una misma persona, se pueden hallar, posiciones y fragmentos de tipo muy distinto, que a veces pueden ser utilizados para mostrar al gún tipo de razonamiento o argumento mientras que otros, pueden senvir para indicar su contrario. Por eso, de la misma manera que no se trata de hacer corresponder un argumento a una persona, tampoco se trata de recoger cuantas personas coinciden en una de estas construcciones.

8. Aun habiendo hecho posible una devolución de resultados y un debate para la elaboración del documento final con l@s participantes y habiendo realizado distintas discusiones sobre los resultados, en distintos momentos de la investigación con compañer@s que participaron en el proyecto. 
dinámicas y variadas, donde se combinan las interacciones con las posiciones de los discursos. Así pues, muchos de los efectos son más bien muestra no sólo de prejuicios y valores, sino que proceden de lo que incorpora la misma psicología, con sus técnicas, sus instrumentos teóricos y prácticos y lo que la fundamenta. Por supuesto, es este último aspecto el más difícil de asumir. Pero es el que más directamente interviene en los efectos autoritarios.

\section{Reflexión sobre algunos resultados sobre feminismo y género en psicología}

Respecto a los resultados, en general, aunque había una buena predisposición hacia el tema "género" o lo que se entendía por "psicología de la mujer", ésta se complicaba bastante en el momento en que se introducía el feminismo ya fuera para la historia de la psicología o para la actualidad. Tanto en las entrevistas como en los grupos de discusión se construyen discursivamente opiniones favorables a las aportaciones de los estudios de género, mientras se combinan intervenciones que desacreditan las feministas como movimiento o como estudiosas. Quiero decir, que podemos encontrarnos con una incorporación o reproducción de algunas de las ideas propuestas desde la voluntad de transformar las relaciones sociales, que se refieren a la igualdad, a los prejuicios o a la discriminación, en la psicología mainstream como señal de progreso pero sin relacionarlo con ellas o abiertamente construyendo una estereotipada imagen del feminismo que es claramente rechazada como generadora de desigualdad hacia los hombres, o de ideológica e interruptora del quehacer científico. Pero, aunque las explicaciones sobre las "peligrosas relaciones académicas" (Cabruja, 2004), están bien documentadas desde la epistemología, la metodología y el activismo feminista como comentaba en la introducción, poseemos pocos estudios que recojan cómo se articulan en la práctica del quehacer psicológico o, también, cuales son las opiniones que dan $1 @_{s}$ estudiantes, $@_{s}$ investigadores y profesores así comol@s profesionales sobre el tema. Un aspecto muy característico de las respuestas era combinar aspectos parciales de las aportaciones feministas pero disociadas radicalmente de sus fuentes, con una dilemática combinación de aceptaciones y rechazos. Voy a comentar algunos de ellos.

\section{Construcciones de la historia de la psicología y la ausencia de mujeres psicólogas: "temporalidad diferencial" e "impersonalidad" como constructos justificativos}

En los resultados de los grupos de discusión y las entrevistas en el momento de recordar mujeres que formaron parte de la historia de la psicología, se produjo una gran coincidencia en que los únicos nombres que se recordaban eran de 
mujeres psicoanalistas. Sin embargo, las explicaciones respecto al porqué esto era así, podían, en cambio, ir en sentidos muy distintos y, por lo tanto, no socavar la imagen de neutralidad de la psicología. Por ejemplo, un recurso en este sentido era aludir a la temporalidad; es decir, como si la causa fuera el desarrollo histórico de los temas de psicología, en el sentido de justificar el recuerdo de mujeres psicoanalistas porque el psicoanálisis es más antiguo y, en cambio, en otros campos más recientes es más difícil que estén. Un efecto es salvaguardar la neutralidad de la psicología y no reconocer su sexismo, sino explicarlo por otro tipo de razones. Una argumentación en este sentido se puede leer en el siguiente fragmento:

Es que, a ver...hay un decalage temporal. Por ejemplo, si hablamos de jurídica en ningún momento se puede comparar con el psicoanálisis. Porque aquí jurídica empezó con la ley del divorcio en los 70. [...] Quiero decir que cuando yo terminé la carrera las aportaciones de las mujeres desde el psicoanálisis eran clarísimas, y en jurídica era imposible que hubiera [...] Claro es que hay un decalage temporal importante.

Tal y como vemos, la justificación es que en psicoanálisis eran "clarísimas", pero en jurídica "imposible que hubiera". Pero ni en una argumentación ni en la otra, se entra en lo que realmente determina la inclusión o no de las mujeres. ¿Porqué en psicoanálisis, sí? Cuando tampoco es tan evidente, con algunas excepciones. ¿Y por qué en jurídica "imposible”.? Es un buen ejemplo de la importancia de la cuestión de hasta qué punto las relaciones e imaginarios patriarcales impregnan la propia psicología como "libre de" cuerpo, sexo y género en sí misma, como hemos visto, pero también respecto a la docencia, la investigación o la práctica profesional con la idea de un desarrollo de progreso, lineal y evolutivo, que se moviliza, también, respecto a otros aspectos.

De forma parecida, en las discusiones grupales y en las entrevistas se reconocen explícitamente las dificultades por discriminación, sociales o roles, en el sentido de aludir a una situación "anterior", en el pasado, de desigualdad social para las mujeres (herencia de las reivindicaciones y argumento socializado). Esta idea compartida, funciona con distintos constructos temporales comparativos entre el pasado y el presente, conformado, a su vez, por una visión evolucionista "moderna" de un progreso lineal. Un presente que se construye como distinto o, a su vez, respecto a los nuevos campos o áreas de la psicología, como con un inicio, "naturalizando" la falta de mujeres y con una imagen de un desarrollo posterior que se reequilibrará. Funciona también como argumentación de contraste. A su vez, aparecen contra argumentos, donde las construcciones comparativas con un presente que se da por supuesto que hay un cambio, vienen matizadas por la experiencia de la práctica laboral, por 
ejemplo. Así, en una intervención, se hace constar la dificultad de que "un hombre vaya a terapia" como más difícil y que "acepte ir con una mujer" (es decir, que la terapeuta sea mujer) aún más o, aunque se afirma que actualmente sí hay mujeres psicólogas en los campos de especialización, en algunas áreas se citan nombres y en otras, no se consiguen recordar.

Otra de las argumentaciones, procede transformando la "asujetización" de la ciencia al revés. Bueno, aparentemente, "al revés”, ya que, en realidad, forma parte del mismo sustrato explicativo y el mismo mecanismo. En este caso se explica la falta de recuerdo de mujeres en la historia de la psicología porque sólo aparecen las iniciales, refiriéndose a las construcciones "impersonalizadas" de los documentos científicos, tal y cómo opera la "retórica de la objetividad" (Cabruja; Vázquez, 1995), con lo cual, al ser neutra, no es posible conocer quien hace las aportaciones, pero dando por supuesto que sus aportaciones han sido incorporadas: "Si bien sus contenidos han sido también tomados en cuenta, no se ha dirigido la atención a si eran mujeres, [...] se citaba una lista de autores haciéndolo invisible". Recoge el argumento feminista de la "invisibilización sexuada" del saber y las "marcas" androcéntricas, a partir de sólo poner las iniciales o de "narrar" en tercera persona o sólo poner los apellidos, pero, justamente para indicar que la neutralidad dificulta saber si hay mujeres. Es decir, para "salvar" la idea de que la psicología no es que no recogiera las aportaciones de las mujeres, sino que no lo sabemos porque no figuran la totalidad del nombre propio y sólo aparecen las iniciales.

\section{Las construcciones del feminismo como causa de "desigualdad" y el género como "equitativo o compensado"}

Los debates sobre los usos y abusos del término género desde documentos institucionales hasta publicaciones especializadas (Tubert, 2003) sirven para entender lo que ha sucedido con una categoría analítica bien polémica en sus usos. La idea es que el feminismo en vez de trabajar para las transformaciones sociales de las relaciones de desigualdad lo que hace es beneficiar a las mujeres y el género en cambio se ocupa de los "dos" o "más", que es una construcción que ha permitido, aunque no lo voy a tratar aquí, la entrada del género, sólo de una determinada manera, en su versión más "suave" y menos comprometida. En las intervenciones aparece construido con una gran diversidad de repertorios. Entre ellos, podemos encontrar desde los que se refieren al feminismo como exagerado, pero sobretodo, la mayoría se apoya argumentativamente, en el desequilibrio de poder o la injusticia o desigualdad que el feminismo puede generar al centrarse en las mujeres y perjudicar a los hombres. En este primer fragmento, podemos leer esta idea de que es "hacia el extremo" y, sobretodo que "se debería trabajar la igualdad". Con la idea implícita que el feminismo 
no la trabaja. Y, que queda desarrollado más adelante con una afirmación que lo ideal es "ni feminismo" ni "machismo". Los dos se colocan como contrapuestos y a un mismo nivel. Como si no estuviera bien el machismo, no es deseable, pero el feminismo tampoco, porque invierte las relaciones de poder. La propuesta es "Yo digo igualdad. No feminismo o machismo", lo cual lleva a imaginar que el machismo va a favor de los hombres y el feminismo a favor de las mujeres, como construcción que se desarrolla a un mismo nivel y no como respuesta al machismo, es decir, abstraida de las condiciones de desigualdad económica, social e histórica:

Encuentro que el feminismo es un poco, más hacia el extremo, bueno, hacia el extremo. Y que se debería de trabajar la igualdad. No feminismo o machismo. Porque entonces se daría una competencia entre hombres y mujeres, más fuerte aún, creo, no? Quiero decir...se trataría de trabajar más la igualdad, que el feminismo o que el machismo...Bueno si lo llevamos al extremo acabaría al revés [...] el que saldría perdiendo es el hombre. Y no es cuestión de que uno pierda y el otro gane. [...] Yo digo igualdad. No feminismo o machismo.

La idea de presentarlas como propuestas opuestas que se hacen corresponder no con la denuncia de una situación estructural de desigualdad económica y social con posiciones sociales producidas por el funcionamiento del sistema sexo-género, sino como lo que "dan" o "procuran" a hombres y mujeres. Un tema que ya posee su propia historia tanto dentro de la ciencia como del feminismo y en teoría política, respecto a la construcción de la igualdad y, respecto al cual, se presentan como claves los trabajos que se centran en las construcciones discursivas y su lugar naturalizando las desigualdades de distinto tipo: sociales, económicas, por razón de sexo, de cultura, etc. (Spink; Spink, 2006) y que participan de las prácticas cotidianas, tanto de la ciencia, como de los medios de comunicación de masas, que producen y reproducen estas ideas al respecto.

Una argumentación producto de la heterosexualidad y deudora de la construcción simbólica del patriarcado y de sus estrategias tanto como de los discursos más recientes neoliberales. Discursos sobre la igualdad y la desigualdad que operan desconectando los orígenes de las denuncias con aspectos parcializados. Así, por ejemplo, se construyen de distintas maneras la idea respecto a que el feminismo, se centra sólo en la "psicología de la mujer" o los derechos de la mujer (en otras intervenciones) y no incluye la del hombre, que debería "equilibrarse". Sucede, de hecho, cómo con la construcción de la memoria histórica o del concepto de democracia (Cabruja; Íñiguez; Vázquez, 2000; Íñiguez; Vázquez, 1995). Así, en el caso del género, el equilibrio, la 
igualdad o una "psicología como más ajustada", como se puede leer en el siguiente párrafo:

Si nos centráramos en lo que es la Psicología de la mujer podríamos caer incluso en una especie de... feminismo, que es la palabra esta que he estado intentando evitar todo el rato. Una especie de feminismo. Y que quizás en una perspectiva de estudios de género, encarando lo que es la Psicología del Mujery la Psicología del Hombre. Pues podríamos quizás, llegar a una posición como más androgénica, una Psicología como más ajustada.

En la gran mayoría de investigaciones se nombra el género como reproducción de la diferencia de variable sexo, diferenciando y superponiendo diferencia anatómico-sexual dualista con diferencias culturales y simbólicas o de roles. Género, no es una categoría que aparezca, (a pesar de que pueda ser enormemente discutida su capacidad para explicar y de qué manera las relaciones patriarcales y androcéntricas) fuera del pensamiento feminista. Han sido los usos los que lo han desprendido de su vínculo con el feminismo, y alargado o bien su uso como variable, igual que podría ser la de diferencia sexual; se asume como paralela a la diferencia sexual, despolitizada, es decir, fuera de algún tipo de relación con algún tipo de perspectiva de análisis y acción feminista o perspectiva comprometida y reproduciendo los dualismos y reificaciones que ya caracterizaron incluso el concepto de "androginia".

La idea de la igualdad y el "derecho a", configura asimismo la base argumentativa de algunas de las razones que se presentan, justamente, para la inclusión de una formación en psicología y género (en sus diversas acepciones tal y como hemos visto) pues, justamente, a partir de las experiencias en la intervención, es desde donde se reclama la necesidad de más herramientas que las que proporciona la psicología o, especialmente, desde $1 @_{S}$ estudiantes, se considera que es un "derecho" el conocer, lo que llaman una "perspectiva más", como un "campo", una "especialidad" que puede ser rentable. El imaginario respecto al feminismo, y así mismo, el imaginario creado por la sociedad occidental, vinculada a la ideología de la modernidad, y, especialmente a la racionalidad científica y androcéntrica, junto con las relaciones de poder entre grupos y colectivos, produce una situación bastante paradójica, en la cual, a partir de un momento, cualquier reivindicación de igualdad, revierte en un temor a una nueva discriminación, en parte efecto de la "ilusión" de movilidad, en parte efecto de la pérdida de poder, de tal manera que, reparar, reestructurar, restaurar o simplemente hacer posible una situación más igualitaria, es percibido, mayoritariamente, como que va a perjudicar a otro grupo de personas, las que antes disponían de estos derechos y legitimidades. 
Los argumentos de "forzar" en vez de "compensar": cuando las construcciones sobre las "identidades" suplantan argumentativamente los mecanismos de exclusión y las prácticas de recuperación

En este sentido, por ejemplo, hablando sobre las mujeres en la historia de la psicología, encontramos que se explicita un rechazo a lo que se asocia con el cumplimiento de "cuotas" y las "dudas" de la cualidad de las aportaciones que forma parte de la mayoría de argumentos respecto al tema de la "discriminación positiva": "nunca he visto que alguien me hablara de mujeres psicólogas a lo largo de la historia de la psicología y, además, me parecería fatal que lo hicieran, como tema específico dentro de la psicología”. De alguna forma, la idea que se cumple con un políticamente correcto o con una discriminación positiva, cuando se ve aparecer personas que no se conocen en las aportaciones de los manuales, pero donde, la impresión que se explicita es la de cumplir con esto sin que haya una razón o, al menos, no sea suficientemente conocida la razón de su incorporación. De hecho, esta se interpreta sólo en base a su pertenencia grupal, pero no debido a la injusticia anterior que se pueda haber cometido como consecuencia de los mecanismos de exclusión y discriminación institucionales y del conocimiento psicológico. Tal y como podemos leer en el siguiente fragmento:

tú ves muy claramente que es un tema de cuotas [...] es decir hay los autores que siempre había salido en todos los manuales y hay una mujer y un señor. Y, dices, a ver, las aportaciones de esta persona son suficientemente importantes como para que figuren aquí Porque a veces tienes la impresión que...cuando lo leía pensaba, bueno, yo no había oído nunca hablar de las aportaciones de este hombre, son bastante importantes. A ver, en función de lo que decía el libro, este manual, a mí no me parecía que tuviera suficiente entidad. [...]

La explicación argumentada en base a que sólo si se "hace una aportación importante, muy importante" pero "no iría a buscar una mujer psicóloga", reproduce la creencia de una acción de compensación por cuotas o de discriminación positiva totalmente descontextualizada de la situación anterior desigual e injusta y como producto de una acción basada solo en la identidad grupal minorizada y no en los mecanismos anteriores de exclusión, injusticia y desigualdad. La idea de "forzar" es muy ilustrativa de la idea de descompensación, o ruptura de lo que constituye una creencia en un funcionamiento "justo" respecto la imparcialidad de la ciencia. Este temor a "forzar", se construye en base a los discursos dominantes sobre la igualdad, la representación, la objetividad y la neutralidad que han actuado invisibilizando 
la violencia institucional de su reproducción, en términos de sexismo y racismo, etnocentrismo y logocentrismo. Si la ciencia es neutra y se constituye por acumulación y relevancia de las aportaciones, parece muy difícil que si alguien hace algo verdaderamente importante, no se lo incluya (y se invisibiliza, tanto para el sexismo como para el racismo de la psicología las consecuencias de los mecanismos institucionales de exclusión y de las lógicas de la racionalidad positivista y de su logocentrismo). Razón por la cual su recuperación no se entiende como "reparación" a la anterior exclusión, por ejemplo. Sino como nueva búsqueda. Y, se construye como "dudosa de calidad" la aportación.

Creo que estas ideas que voy a llamar, de suplantación de la actividad por la identidad, y la sospecha de "forzar" en vez de reparar una desigualdad, generan distintas manifestaciones: dudas de la calidad o capacidad e impresiones de que para hacer esta reparación se daña o perjudica a otros; puesto que la inclusión o reparación de una injusticia, histórica, no obedece a una compensación abstracta. Por supuesto, que el fundamento es la creencia en la justicia en la sociedad y en la ciencia y en la enorme dificultad en hacer explícitos los mecanismos de exclusión institucional que construyen una historia de posiciones y lugares de dominación hegemónicas, mientras excluyen a otros.

Pero, a su vez, puede ser que la cotidianidad lleva a experiencias y razonamientos de otro tipo. Sirva como ejemplo que en una de las intervenciones se cuenta que a partir de un trabajo de instituto realizado por adolescentes, se descubre una mujer psicóloga con cargos institucionales y méritos bien demostrados, que no se conoce y que cuando se intenta localizar en los manuales de psicología no aparece a pesar de haber hecho aportaciones muy importantes. Por supuesto que la observación al respecto no deja lugar a dudas de la sorpresa de tener una formación en psicología, no conocer a alguien bastante importante y en el momento de querer realizar su búsqueda, encontrarse con un vacío de citación. Tal y como se puede leer en el siguiente fragmento, la constatación da cuenta de los procesos por los cuales una historia mainstream puede no incorporar y marginalizar una aportación valiosa, ahora recuperada, pero silenciada:

Claro la sorpresa es cuando descubres que es una persona que ha hecho aportaciones importantes, pero la sorpresa mayor es cuando vas a consultar manuales o libros de historia de la psicología y esta autora no estaba.

\section{Conclusiones}

Resumiendo un poco las reflexiones sobre los resultados, creo que aparecen lo que han constituido las grandes peticiones y luchas feministas tanto en lo 
social como en la ciencia, pero desconectadas de su origen, fragmentadas y disociadas de su producción. De hecho, hay una dinámica construcción discursiva que tiende a reificar las relaciones de poder existentes tanto respecto a la diferencia sexual como a la diferencia en la concepción de ciencia. En este sentido hay un efecto de "neutralización" de los trabajos y perspectivas feministas, pues, consecuentemente, lo que se erige como preferido para el aprendizaje y para la investigación es una especia de "Perspectiva de género neutra". Menos amenazante para los fundamentos de la ciencia positivista: el estatus quo, la ciencia correcta, el patrimonio de la ciencia verdadera. Pero también por la disociación entre un concepto abstracto, descontextualizado y neutro de igualdad, respecto a las condiciones de desigualdad y marginación silenciadas, en unas dinámicas relaciones de poder entre grupos El tokenismo como mecanismo que Erika Apfelbaum (1979) explicó tan acertadamente para las relaciones de poder y dominación intergrupales, actúa de la misma forma, también en la ciencia psicológica respecto al feminismo y el género tal y como planteé (Cabruja, 2003). Y esta imagen es la que se construye en las argumentaciones de $@_{s}$ investigadores y psicólog@s en el sentido de creer que participan de un sistema de ciencia justo e igualitario, en el cual, en base a su neutralidad y objetividad, quien es "realmente valioso" triunfa y es incorporado en su historia y quién no, no, así como una idea del evolucionismo y el progreso moderno, respecto a los cambios, argumentada en base a comparaciones temporales y otras de contraste. Así mismo, este imaginario respecto al funcionamiento, equitativo, objetivo, neutral, etc. del conocimiento científico, genera la idea, que se incorporan representantes de grupos minorizados o marginalizados, en base a su "identidad" y no a su "actividad" (cuando la "identidad" suplanta la "actividad" en vez de entenderlo como reparación de un mecanismo de exclusión social e institucional anterior), puesto que ello permite mantener la idea del valor en la calidad y la sospecha continua hacia el valor de lo devaluado así como la minusvalorización de las condiciones de segregación institucional que han permitido que no llegaran a incorporarse. A su vez, la práctica y la cotidianidad profesional, introducen aspectos que contradicen y cuestionan estas mismas posiciones, en la línea de conectar lo académico y lo político, e incluir las desigualdades sociales, económicas y culturales junto con un análisis más crítico de la producción del conocimiento psicológico, tal y como lo conocemos.

Creo que los feminismos siempre han conllevado, de una manera u otra, utilizando el término en un sentido amplio, una acción "deconstructora", hacia las organizaciones del saber, de la sociedad de las relaciones, pero, por supuesto, de maneras muy distintas. Y lo que supone un feminismo deconstruccionista, postmoderno, o posestructuralista, con todas sus diferencias, es que comparte, 
además, una crítica bastante fundamental a los sustratos de la racionalidad positivista, a sus mismas bases, por lo tanto, no es recuperar lo silenciado, o restaurar la igualdad prohibida, sino transformar profundamente las coordenadas fundamentales de la construcción de saber.

Por esta razón, el análisis y problematización que realizo, no descansan en un análisis cualitativo, sino que incorpora, de alguna manera, los intentos para entender y explicar, de otra manera, lo que rodea, acompaña y posibilita las intervenciones, afirmaciones, etc. La crítica feminista en la psicología, sin embargo, no se centra sólo en dirigir la atención a las experiencias de las mujeres o la segregación imperante en las universidades, sino que intenta también desarrollar investigación y práctica no sexista, que incluyen la corrección del sesgo androcéntrico en teorías y métodos o en interpretaciones de conclusiones y a desarrollar la autoreflexividad como método o aplicar la deconstrucción a las creencias producidas desde la propia psicología empleando la elección de temas, hipótesis y métodos para abordar una supuesta diferencia en un sentido u otro, alejada de una psicología neutra, apolítica, descorporeizada y acontextual. Es decir, deconstruir las naturalizaciones y dualismos e incorporar un análisis de género comprometido ética y políticamente con una genealogía de la epistemología y los movimientos feministas, ya sea para los estudios sobre las subjetividades, las sexualidades, y las otredades, donde el heterosexismo y el etnocentrismo, con todas sus intersecciones, especialmente en el contexto de la globalización, dificulten que cuando el "género entre por la puerta...del aula, del despacho, de casa o salga a la calle, el compromiso político con las relaciones de poder no salte por la ventana” contribuyendo, además, a romper, uno más de los dualismos, el de profesionalismo/despolitización.

\section{Referencias bibliográficas}

ANZALDUAA, G. Movimientos de rebeldia y las culturas que traicionan. Borderlands/La Frontera. The New Mestiza, SanFrancisco, Aunt Lute Books, 1987.

BHAVANANI, K-K; PHOENIX, A. (1994) Shifting Identites/Shifting Racisms. A Feminism and Psychology Reader. Vol 4 (1). Sage (London, Thousand Oaks and New Delhi).

Bhavnani, K-Ky Phoenix, A. (ed) 1994. Shifting Identities/Shifting Racisms. A feminism ér Psychological Reader. Feminism é Psychology, 4(1). 1994. Sage

BILLIG, M. et al. Ideological Dilemmas: a social psychology of everyday thinking. London: Sage, 1988.

BURMAN, E. Feminists and psychologicalpractice. London: Sage, 1990.

CABRUJA, T. Género y valores: su conjugación en las teorías de la identidad y las relaciones de género desde una perspectiva deconstruccionista. In: IZQUIERDO, M. Jesús (coord.). Género y valores. Vitoria: Emakunde - Instituto Vasco de la Mujer, 1995, p. 99-123. 
CABRUJA, T.; VAZZQUEZ, F. Retórica de la objetividad. Revista de Psicología Social Aplicada, v.5, n.1/2, Universidad de Alicante, Alicante, p.113-126, 1995.

CABRUJA, T. Psicología social crítica y posmodernidad. Anthropos, Psicología social. Una visión crítica e histórica, n.1, 177, Barcelona, marzo-abril, 1998.

CABRUJA, T.; IÑÍGUEZ, T.; VÁZQUEZ, F. ¿Cómo construimos el mundo? Relativismo, espacios de relación y narratividad. Anàlisi. Quaderns de comunicació i cultura., v.25, Barcelona, p.61-94, 2000.

CABRUJA, T.; GORDO, A. The (un)state of Spanish critical psychology. The International Joumal of Critical Psychology, v.1, London, p.128-135, 2001.

CABRUJA, T. Astucias de la razón y psicología crítica: condiciones de crotismo-seducción, prácticas de tokenismo y resistencias ético-políticas. Política y Sociedad, A partir del socio construccionismo. Madrid, v.40, n.1, p.141-153, 2003.

CABRUJA, T. et al. Com incorporar les dones psicòlogues i els estudis feministas a la formació psicologica? Estudi pilot a Girona i comarques. Informe. Texto mecanografiado, diciembre 2004.

CABRUJA, T. Feminismo y Psicología: Relaciones académicas/Relaciones peligrosas. III Encuentro de Iniciativas Criticas en Disciplinas "Psi". Psicología, poder y sociedad. Texto mecanografiado. 2004.

CABRUJA, T.; ESPINAS, E.; ALBERTIN, P.; JUANOLA, E.;TORRENT, A. ¿Cómo incorporar las mujeres psicólogas y los estudios feministas a la formación psicológica? Análisis de las producciones discursivas de psicólog@s en formación y profesionales. In: MARTÍNEZ, J. Romay; MIRA, R. García (eds.). Psicología social y problemas sociales. Psicologia juridica, de la violencia y de género (p.437-444). Madrid: Biblioteca Nueva, 2005.

CALLAGHAN, J. Becoming a psychologist: professionalism, feminism, activism. Feminisms ó Activisms. Annual Review of Critical Psychology. Issue 4, p 154-165, 2005.

CAPDEVILA, R.; UNGER, R. Feminisms without borders: exploring the relationships between feminist and political psychology. Feminism of Psychology, v.16 (1), p.5-11. London: Sage, 2006.

CÓRDOBA, D.; SÁEZ, J.; VIDARTE, P. Teoria Queer. Editorial: Egales. Editorial: Gai y Lesbiana. 2.ed., 2005.

COULSON, M.; BHAVNANI, K-K. Making a difference-questioning women's studies. In:. BURMAN, E. Feminists and psychological practice. London: Sage, p. 62-75, 1990.

CRESSY, E.; HARRICK, E.; FUEHRER, A. The narrative study of feminist psychological identites. Feminism \& Psychology, v.12(2), p.221-246, London: Sage, 2002.

DAVIES; HARRÉ. Positioning: the discoursive production of selves. Journal of the Theory of Social Behaviour, v.20, p. 43-65, 1990.

FERNANDEZ-CHRISTLIEB, P. (1994). La psicología colectiva un fin de siglo más tarde. Barcelona: Anthropos. 
FIGUEROA-SARRIERA, H.; MILAGROS LÓPEZ, M.; ROMÁN, M. (Eds.). Más allá de la bella (in) diferencia: revisión postfeminista y otras escrituras posibles. San Juan: Pub. Puertorriqueñas, 1994.

GARCIA DAUDER, S. Psicologia y feminismo: historia olvidada de mujeres pioneras en Psicología. Madrid: Narcea, 2005.

GARCIA-COLMENARES, C. Autoridad femenina y reconstrucción biográfica: el caso de las primeras psicólogas españolas. Revista de investigación en educación. n.3, p. 51-70, 2006.

GORDO, A. De la crítica al academicismo metodológico: Líncas de acción contra los desalojos sociocríticos. Athenea Digital, n.1, 2002. Revista de pensamiento e investigación social, 2002.

HARDING, S. The science question in feminism: Milton Keynes. Open University Press, 1986. HARE-MUSTIN, R.; MARECEK, J. Marcar la diferencia. In: HARE-MUSTIN, R. Y.; MARECEK, J. Marcar la diferencia: psicología y construcción de los sexos. Barcelona: Herder, p. 15-38, 1990.

HENWOOD, K.; GRIFFIN, C.; PHOENIX, A. Standpoints and differences: essays in the practice of feminist psychology. London: Sage, 1998.

HOLLWAY, W. Subjectivity and method in psychology: gender, meaning and science. London: Sage, 1989.

HOOKS, Bell. Feminist Theory: from margins to center. Boston: South End Press, 1984.

IBÁNEZZ, T. Psicología social construccionista. México: Universidad de Guadalajara, 1994.

IÑIGUEZ, L.; VAZZQUEZ, F. Legitimidad del sistema democrático. Análisis de un discurso autorreferencial. In: D'ADAMO, O.; GARCÍA, V.; MONTERO, M. (Comps.). Psicología de la acción política. Buenos Aires: Paidós, 1995.

INÍGUEZ, L. Análisis del discurso: manual para las ciencias sociales. Barcelona: Ediuoc, 2003.

INÍGUEZ, L. Nucvos debates, nuevas ideas y nuevas prácticas en la Psicología social de la era "post-construccionista". Athenea Digital, Revista de pensamiento e investigación social, n.8, 2005.

KAGAN, C.; LEWIS, S. Where's your sense of humour? Swimming against the tide in higher education. In: BURMAN, E. Feminists and psychologicalpractice. London: Sage, p.18-32, 1990.

KITZINGER, C. Resisting the discipline. In: BURMAN, E. Feminists and psychological practice. London: Sage, p. 119-139, 1990.

LAGARDE, M. Claves feministas: cuadernos inacabados. Madrid: Horas y horas, 2001.

MARTIN BARO, I. (1987) El reto popular a la psicología social en América Latina. En Boletín de Psicología, UCA, VI (26) 251-270.

MONTERO, M. (Coord.). Construcción y critica de la psicología social. Barcelona: Anthropos, 1994.

PARKER, I. Dyscoure dymamics: critical analysis for social and individual psychology. Londres: Routledge, 1992. 
POTTER, J. La representación de la realidad. Discurso, retórica y construcción social. Barcelona: Paidós, 1996.

RAMAZANOGLU, C.; HOLLAND, J. Feministmethodology: challenges and choices. London: Sage, 2002.

SPINK, P.; SPINK, M.J. Práticas cotidianas de naturalização da desigualdade. São Paulo: Cortez Editora, 2006.

SPIVAK, G. Can the subaltern speak? In: NELSON, C.; GROSSBERG, L. Marxism and the interpretation of cultura. London: McMillan, 1988.

STOLKE, Verena. “QQué revolución feminista en la enseñanza?: Estudios de la Mujer o Integración curricular”. Papers, Revista de Psicología. Barcelona: UAB, p.33-42, 1988.

UNGER, R. Resisting gender: twenty-five years of feminist psychology. London: Sage, 1998.

WETHERELL, M.; POTTER, J. Mapping the language of racism: discourse and the legitimation of explotation. Brighton: Columbia University Press, 1992.

WILKINSON, C.; KITZINGER, C. Feminism and discourse: psychological persepctives. Londres: Sage, 1995.

Recebido em maio de 2007 e aprovado em agosto de 2007. 